\title{
Diabetic Retinopathy Environment-Wide Association Study (EWAS) in NHANES 2005-2008
}

\author{
Kevin Blighe $^{1}\left(\mathbb{D}\right.$, Sarega Gurudas ${ }^{1}$, Ying Lee ${ }^{1}\left(\mathbb{D}\right.$ and Sobha Sivaprasad ${ }^{1,2, *(\mathbb{D})}$ \\ 1 Institute of Ophthalmology, University College London, London EC1V 9EL, UK; k.blighe@ucl.ac.uk (K.B.); \\ sarega.gurudas.17@ucl.ac.uk (S.G.); ying.lee@tu-dresden.de (Y.L.) \\ 2 National Institute for Health Research, Moorfields Biomedical Research Centre, London EC1V 2PD, UK \\ * Correspondence: sobha.sivaprasad@nhs.net; Tel.: +44-(0)20-7566-2039
}

Received: 1 October 2020; Accepted: 9 November 2020; Published: 12 November 2020

\begin{abstract}
Several circulating biomarkers are reported to be associated with diabetic retinopathy (DR). However, their relative contributions to DR compared to known risk factors, such as hyperglycaemia, hypertension, and hyperlipidaemia, remain unclear. In this data driven study, we used novel models to evaluate the associations of over 400 laboratory parameters with DR compared to the established risk factors. Methods: we performed an environment-wide association study (EWAS) of laboratory parameters available in National Health and Nutrition Examination Survey (NHANES) 2007-2008 in individuals with diabetes with DR as the outcome (test set). We employed independent variable (feature) selection approaches, including parallelised univariate regression modelling, Principal Component Analysis (PCA), penalised regression, and RandomForest ${ }^{\mathrm{TM}}$. These models were replicated in NHANES 2005-2006 (replication set). Our test and replication sets consisted of 1025 and 637 individuals with available DR status and laboratory data respectively. Glycohemoglobin (HbA1c) was the strongest risk factor for DR. Our PCA-based approach produced a model that incorporated 18 principal components (PCs) that had an Area under the Curve (AUC) 0.796 (95\% CI $0.761-0.832$ ), while penalised regression identified a 9-feature model with $78.51 \%$ accuracy and AUC 0.74 (95\% CI 0.72-0.77). RandomForest $\mathrm{t}^{\mathrm{TM}}$ identified a 31-feature model with 78.4\% accuracy and AUC 0.71 (95\% CI 0.65-0.77). On grouping the selected variables in our RandomForest ${ }^{\mathrm{TM}}$, hyperglycaemia alone achieved AUC 0.72 (95\% CI 0.68-0.76). The AUC increased to 0.84 (95\% CI 0.78-0.9) when the model also included hypertension, hypercholesterolemia, haematocrit, renal, and liver function tests.
\end{abstract}

Keywords: environment wide association study; diabetic retinopathy; hyperglycaemia; circulating biomarkers

\section{Introduction}

Diabetic retinopathy (DR), a chronic diabetes complication, is generally believed to be the most common cause of microvascular changes in the retina. The initial retinal lesions of diabetic retinopathy (DR) are microaneurysms, but they can occur in eyes with and without diabetes [1-3]. With increasing duration of diabetes, other lesions develop and co-exist in the retina, such as retinal haemorrhages, exudates, intraretinal microvascular abnormalities, and neovascularization of the retina or optic disc. Based on the presence of individual lesions or a constellation of them, DR severity level is graded from mild, moderate, and severe non-proliferative diabetic retinopathy (NPDR) to proliferative diabetic retinopathy (PDR) [4,5]. Diabetic macular oedema (DME) can occur in any stage of DR [5]. In population-based studies, approximately a third of people with diabetes have DR [6,7].

The established systemic risk factors for DR are suboptimal control of hyperglycaemia, hypertension, and hyperlipidaemia [8,9]. Hypertension can also cause some of these retinal lesions independent of diabetes [10]. There are several laboratory parameters that have been shown to be 
abnormal in people with DR, such as hyperuricemia [11], low vitamin D levels [12], low thyroxine levels [13], anaemia [14], oxidative stress, and inflammatory markers [15]. In addition, DR is also associated with markers of diabetic kidney disease, including microalbuminuria and serum creatinine [16,17], and cardiovascular disease markers, such as raised C-reactive protein (CRP) [18]. Most of these associations and risks of DR are reported based on analysis of candidate laboratory-based serum or urinary markers.

In addition to these risk factors, there are several other non-modifiable and modifiable risk factors that have been attributed to the development and progression of DR. Some of these include age of onset of diabetes, duration of diabetes, male sex, and ethnicity [19-21]. However, the relative associations of these risk factors and circulating biomarkers with DR is unclear.

There is an unmet need to rank these reported retinal, systemic (blood pressure, body mass index) risk factors, and laboratory markers so that preventative measures may systematically target modifiable factors and markers. As DR is a multifactorial complication of diabetes involving several metabolic and biochemical pathways, ranking will inform clinical practice on how to systematically evaluate and control appropriate risk factors or biomarkers based on a rank order. The ranking may also identify new druggable targets.

The National Health and Nutrition Examination Survey (NHANES-https://wwwn.cdc.gov/Nchs/ Nhanes/) was initiated in the 1960s in order to examine the health and nutritional status of U.S. citizens. Since 1999, it has examined approximately 5000 citizens per year, and the data includes diabetes, eye diseases, and several laboratory markers, including environmental toxins, allergens, and pollutants.

In this study, we used an environment wide association study (EWAS) methodology [22-25] on NHANES 2007-2008 to evaluate the rank order of systemic and laboratory risks of DR among individuals identified as having diabetes to evaluate their relative associations with DR. Our findings were then replicated in NHANES 2005-2006. Our objective is not to only use previously reported risk factors, but also provide new research avenues from this data-driven model-agnostic study.

\section{Methods}

\subsection{Study Data Collection}

We used National Health and Nutrition Examination Survey (NHANES) 2007-2008 as our primary cohort and 2005-2006 as a replication cohort. Both datasets were prepared in the same fashion; however, for ease of interpretation, the following methods describe 2007-2008.

\subsection{Data Collection}

Specifically, three main categories of data were used: examination data (Ophthalmology—Retinal Imaging data), demographics data, and laboratory data. Demographic data included age, sex, and ethnic origin. Socioeconomic variables included education, marital status, and family income.

\subsection{Diabetes Status}

Definition of diabetes: the selection criteria for diabetes included self-reported diabetes, on anti-diabetes drugs, taking insulin, fasting blood sugar (FBS) $\geq 6.1(110 \mathrm{mg} / \mathrm{dL})$, random blood sugar $(\mathrm{RBS}) \geq 11.1(200 \mathrm{mg} / \mathrm{dL})$, oral glucose tolerance test $(\mathrm{OGTT}) \geq 200 \mathrm{mg} / \mathrm{dL}$, glycohemoglobin $(\mathrm{HbA} 1 \mathrm{c}) \geq 6.5 \%$.

\subsection{Diabetic Retinopathy}

The NHANES cohort underwent fundus photography using the Canon CR6-45NM ophthalmic digital imaging system and Canon EOS 10D digital camera (Canon, Tokyo, Japan). Two digital images per eye were captured through a non-pharmacologically dilated pupil, one centred on the macula and the second on the optic nerve. The digital images were graded by masked photo graders at the University of Wisconsin Ocular Epidemiologic Reading Center, Madison, using a modification 
of the Airlie House classification system. The main outcome of interest in the examination data was " 4 levels retinopathy severity, worse eye"-this variable was recoded as binary with levels: no retinopathy; retinopathy (including mild non-proliferative retinopathy (NPR), moderate/severe NPR, and proliferative).

\subsection{Covariates}

Age, ethnicity, and diabetes duration were used as covariates. Diabetes duration was calculated as age at screening, minus the age at which the individual was first informed that he/she had diabetes.

\subsection{Statistical Analysis}

Prior to any analysis, in addition, any variable that contained a single value occupying more than $90 \%$ of total values was removed, as were variables that had more than $90 \%$ missingness. Further specific filtering and encoding was then applied per dataset. (A) Examination data: variables that were different encodings of the main outcome were removed; variables related to the status of the examination appointment were removed; variables related to glaucoma, for which there is already a single variable, were removed; variables related to the left or right eye where there was already a variable for 'worse' eye were removed; values encoded as missing were recoded as not available; and all other remaining variables were encoded as binary, with 0 representing the absence of the condition, and 1 representing any recorded presence (at any level) of the condition. (B) Demographic data: variables associated with interpreters and the language of the interview were removed; variables that were duplicates or different encoding of each were removed. (C) Laboratory data: categorical variables were removed and only continuous retained; duplicate variables related to the oral glucose tolerance test were removed; variables related to time since domestic activities ("pump gas", "shower", etc.) were removed; variables that were duplicates or different encodings of each were removed; variables measured on the imperial system of weights and measures were removed if they had a corresponding variable in SI units. We focused only on continuous laboratory variables for the following reasons: 1, in NHANES, the majority of categorical variables are derived from the continuous variables; 2, our Principal Component Analysis (PCA)-based approach can only work on continuous variables; 3, for RandomForest ${ }^{\mathrm{TM}}$, having continuous variables increases the number of splitting points in the data, and metrics of importance such as Gini are known to exhibit less bias on such data [26].

All datasets were downloaded as SAS XPORT (xpt) format and read into R (v4.0.2) via the Hmisc package.

Individuals with a missing value in the main outcome variable were removed before aligning the examination, demographics, and laboratory data via each individual's respondent sequence number (SEQN). This dataset was then further filtered for only those individuals who had diabetes (Figure 1). Variables were removed from the data that had 0 variance (i.e., constant values) (Supplementary Table S1).

Prior to statistical analysis, continuous laboratory variables were $\operatorname{logged}\left(\log _{\mathrm{e}}\right)$ and then transformed into z-scores to ensure that these were on the same scale. In regression analysis, the complex sampling design of the NHANES dataset was accounted for through use of survey sampling weights via the survey package in R/CRAN. To do this, the following value-pairs were used with the svydesign function: (id, SDMVPSU; strata, SDMVSTRA; weights, WTMEC2YR; nest, TRUE).

Univariate analysis was performed on all candidate predictors using a survey-weighted compute-parallelized logistic regression model via the R/Bioconductor package RegParallel, adjusting for age, ethnicity, and duration of diabetes separately. The Benjamini-Hochberg [27] procedure was used to control the type I error false discovery rate (FDR). A customized Manhattan plot was generated using ggplot2, while pairwise scatter and correlation plots were generated via a customized pairs plot. Finally, a heatmap was generated via the R/Bioconductor package ComplexHeatmap.

As our study is also hypothesis-generating, multivariate approaches based on principal component analysis (PCA), penalised regression, and the RandomForest ${ }^{\mathrm{TM}}$ classification algorithm were additionally used. Variables were pre-filtered and prepared as per univariate testing. Principal component analysis 
was performed via the R/Bioconductor package PCAtools. After conducting PCA, each eigenvector was then independently regressed against retinopathy outcome via binary logistic regression and those that passed $p \leq 0.05$ were used to construct a multivariable model that was further tested in ROC analysis via the $p R O C$ package in $\mathrm{R}$.

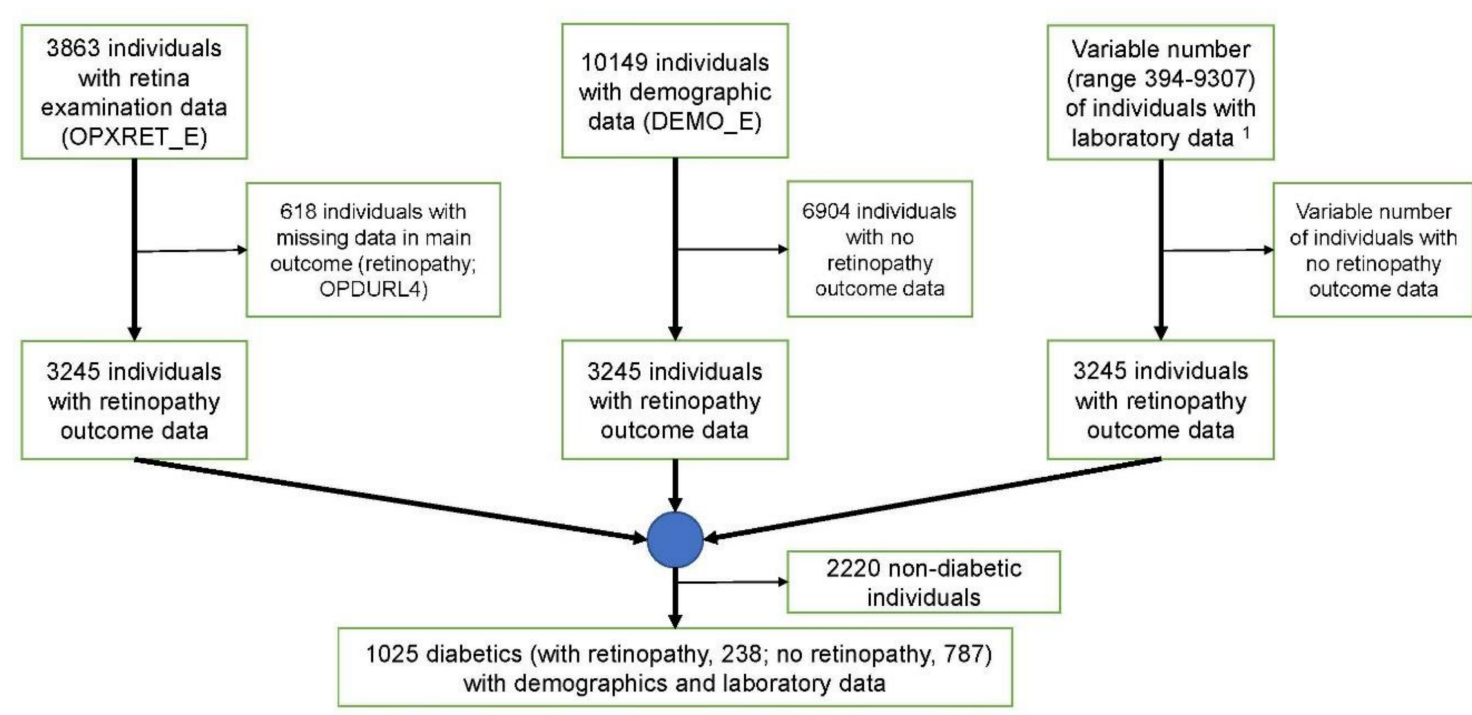

Footnotes:

1 Laboratory datasets (2007-8): ALB_CR_E; APOB_E; BIOPRO_E; BPX_E; CARB_E; CBC_E; COTNAL_E; CRP_E; DEET_E; ENX_E; EPH_E; FASTQX_E; FERTIN_E; FOLATE_E; FOLFMS_E; GHB_E; GLU_E; HDL_Ë; HEPA_E; HEPBD_E; HEPB_S_E; HEPC_E; HIV_E; HPVSER_E; HPVSWR_E; HSV_E; IHG_E; OGTT_E; OPD_E; PAH_E; PBCD_E; PERNT_E; PFC_E; PHTHTE_E; PHYTO_E; POOLTF_E; PP_E; PSA_E; SSHCV_E; SSUSG_E; TCHOL_E; TFR_E; THYROD_E; TRIGLY_E; UAM_E; UAS_E;

UCPREG_E; UHG_E; UHM_E; UIO_E; UPHOPM_E; UPP_E; VID_E; VIT_B6_E; VOC_E; VOCMWB_E; VOCWB_E.

Figure 1. Cohort selection process for the National Health and Nutrition Examination Survey (NHANES) 2007-2008.

Separately, as model complexity and multi-collinearity can arise from a large number of predictors, elastic net regularization (penalised regression with L1 and L2 penalties of the Lasso and Ridge methods) was used to reduce the number of predictor variables using glmnet in R/CRAN. To fit the model, 100x cross-validation was used and alpha $(\alpha)$ set to 0.5 . The final chosen variables were those whose coefficients were not shrunk to zero-these were plot as violin plots with scatter overlays to show differences between non-DR and DR via ggplot2. To determine accuracy, model predictions were made on the data using the lambda $(\lambda)$ one-standard-error rule using the predict function from the stats package in $\mathrm{R}$.

Finally, the RandomForest ${ }^{\mathrm{TM}}(\mathrm{RF})$ model was fitted via the randomForest $\mathrm{R} / \mathrm{CRAN}$ package. For this, the dataset was divided randomly into 50\% training and 50\% validation. Prior to model fitting, the initial model was tuned using functionality provided by the caret package in R/CRAN, as follows: (1), a 10x cross-validation control function was defined via trainControl function; (2) the best value for 'mtry', i.e., the ideal number of variables to randomly sample, was determined using the train function across a search/tuning grid ranging between 1-40 and with Kappa as the metric; and (3) using the selected value of 'mtry', the ideal number of trees, 'ntrees', was determined also via the train function with selection metric based on Kappa. After the initial model was fit, variables with mean decrease in accuracy $\leq 1 \%$ were excluded and the model re-fit. This was then repeated in a recursive fashion until all variables with negative mean decrease accuracy were removed from the model.

\subsection{Final Risk Models}

Variables selected from RandomForest ${ }^{\mathrm{TM}}$ were grouped based on similarity of function or clinical use. Each group was then used to create independent univariate or multivariable binary logistic regression models with DR as the end-point. A single Wald test $p$-value was derived for each model 
using wald.test from the aod package. ROC analysis was performed using $p R O C$. McFadden's and Nagelkerke's pseudo- $R^{2}$ were derived via the $p s c l$ and $r m s$ packages, respectively.

\section{Results}

\subsection{Study Cohort}

In NHANES 2007-2008, retinal imaging data is available for 3863 individuals, demographics data is available for 10,149 individuals, and laboratory data is available for between 394 and 9307 individuals, depending on the individual laboratory dataset in NHANES (see Figure 1 footnote). After aligning all data and filtering for those who had diabetes by our classification, 1025 individuals remained in our dataset. The selection process is illustrated in Figure 1, while Table 1 provides an overview of the demographics of these individuals.

For our replication cohort, NHANES 2005-2006, we prepared laboratory data following the same filter criteria as NHANES 2007-2008 and produced a final dataset of 2459 individuals, among which 637 (with retinopathy, 176; no retinopathy, 461) had diabetes.

\subsection{Retinal Lesions of Diabetic Retinopathy}

To help validate our methodology and cohort selection, we aimed to determine retinal lesions that define DR. To this end, we identified nine retinal lesions in NHANES 2007-2008 that were statistically significantly associated with DR and survived to $p$-value adjustment for false discovery (Table 2). The top lesions were retinal microaneurysms ( $p \leq 0.0001)$, followed by retinal hard exudates (typically due to lipoprotein deposition in the retina and may be associated with macular oedema) $(p \leq 0.0001)$. Other key lesions at $p \leq 0.0001$ were retinal soft exudate (now termed cotton wool spots), retinal blot haemorrhages, intraretinal microvascular abnormalities (IRMA), and macular oedema. In NHANES, retinal microaneurysms and retinal blot haemorrhages are encoded to be mutually exclusive, i.e., an individual is recorded as having retinal microaneurysms only when not accompanied with retinal blot haemorrhages, and vice-versa (Table 2).

\subsection{Univariate Logistic Regression Analysis}

In total, six variables reached statistical significance in the unadjusted univariate analysis, 11 after adjustment for age, two after adjustment for ethnicity, and seven for diabetes duration (Supplementary Figure S1; Table 3). Glycohemoglobin ( $\mathrm{HbA1c}$ ) was the only variable that was statistically significant in both the unadjusted and adjusted analyses. Other risk variables of note that reached statistical significance in the unadjusted analysis included serum glucose (mmol/L) (i.e., RBS), osmolality $(\mathrm{mmol} / \mathrm{Kg})$, urinary albumin $(\mathrm{mg} / \mathrm{L})$, and fasting glucose $(\mathrm{mmol} / \mathrm{L})$ (i.e., FBS). The only protective variable, i.e., negatively associated, was haemoglobin $(\mathrm{g} / \mathrm{dL})$. These variables indicate suboptimal diabetes control, abnormal kidney function, and presence of anaemia as risk factors for DR. There was evidence of co-correlation among these statistically significant variables from the unadjusted analysis (Supplementary Figure S2).

Interestingly, after adjustment for diabetes duration, the following variables reached statistical significance: $\mathrm{HbA1c}(\%)$, osmolality $(\mathrm{mmol} / \mathrm{Kg})$, urinary iodine $(\mu \mathrm{g} / \mathrm{L})$, urinary cobalt $(\mu \mathrm{g} / \mathrm{L})$, urinary triclosan $(\mathrm{ng} / \mathrm{mL})$, urinary creatinine $(\mu \mathrm{mol} / \mathrm{L})$, and urinary barium $(\mu \mathrm{g} / \mathrm{L})$.

\subsection{Principal Component Analysis}

Unsupervised PCA using all laboratory variables revealed that 59 PCs could account for $80 \%$ or more variation in the dataset. Eighteen PCs were statistically significantly associated with DR at $p \leq 0.05$ via independent binomial regression models testing each PC (Supplementary Table S2). The top variables responsible for variation along these PCs included measures of blood glucose (HbA1c, random blood glucose and fasting blood glucose), kidney function markers (urinary albumin, blood urea nitrogen (BUN)), haematological markers (haematocrit, haemoglobin, red blood cell distribution 
width), inflammatory markers (CRP), white blood cell count, urinary nitrates, segmented neutrophil count), and toxic elements (urinary beryllium and cotinine) among others-these PCs were also statistically significantly correlated to microaneurysms, the previously-identified top retinal lesion, and the covariates used during univariate testing (Supplementary Figure S3). Through ROC analysis, these 18 PCs achieved an Area Under the Curve (AUC) of 0.796 (95\% CI: 0.761-0.832).

\subsection{Penalised Regression Model}

We fitted an unbiased elastic-net penalised regression model to the laboratory variables and cross-validated it 100x. The model selected nine variables whose coefficients were not shrunk to zero: urinary albumin, BUN, urinary cobalt, $\mathrm{CRP}, \mathrm{HbA1c}$, blood osmolality, serum potassium, systolic blood pressure, and urinary nitrate (Figure 2). Of note, these measurements mainly represent diabetes and blood pressure control and kidney function. This model had an accuracy of $78.51 \%$ and AUC 0.74 (95\% CI: 0.72-0.77) when predicted on the same dataset on which the model was produced.

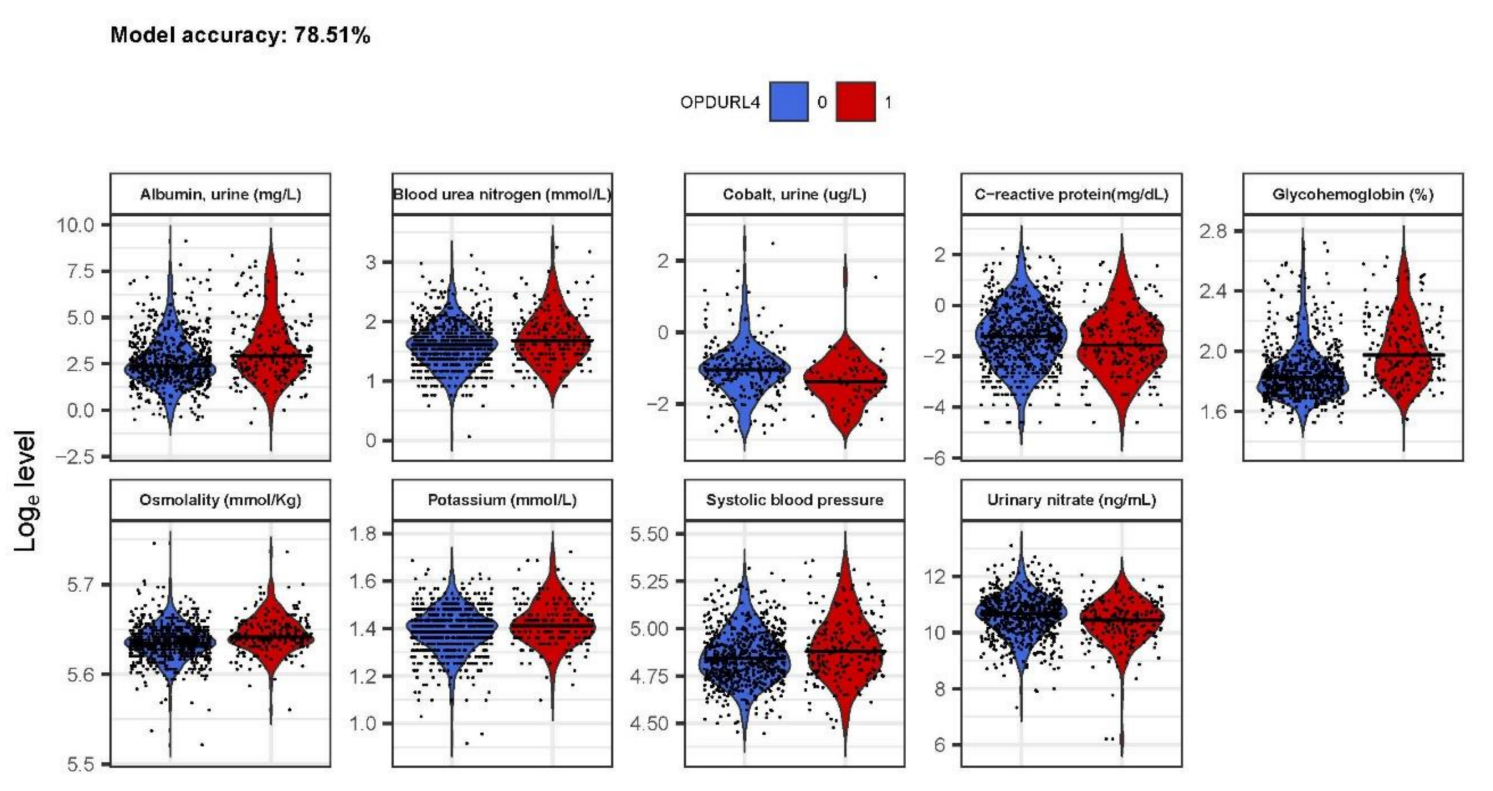

Figure 2. Penalised regression-selected variables. Variables were selected from a $100 \times$ cross-validated model with $\alpha=0.5$. Final variable selection was based on coefficients not shrunk to 0 . Model accuracy was determined to be 78.4\% accuracy and AUC 0.71 (95\% CI: 0.65-0.77).

\subsection{Randomforest ${ }^{\mathrm{TM}}$ Classification Model}

From our RandomForest ${ }^{\mathrm{TM}}$ model, $\mathrm{HbA1c}$ was the single best predictor of DR (mean decrease accuracy, 31.94\%; Gini, 21.75) (Table 4). However, other notable variables of appreciable accuracy were markers of diabetes control (FBS, RBS) inflammation (CRP), kidney function (potassium, BUN, creatinine and urinary albumin), haematological markers (haematocrit), and systolic blood pressure, among others. The overall accuracy of the model on the validation cohort was $78.4 \%$ and AUC 0.71 (95\% CI: 0.65-0.77). 
Table 1. Demographic overview of study cohort.

\begin{tabular}{|c|c|c|c|c|c|c|}
\hline $\begin{array}{c}\text { Characteristics } \\
\text { Mean }( \pm \mathrm{SD}) \\
n(\%)\end{array}$ & & $\begin{array}{c}\text { Diabetes with } \\
\text { Non-Diabetic } \\
\text { Retinopathy } \\
(n=787)\end{array}$ & $\begin{array}{c}\text { Diabetic } \\
\text { Retinopathy } \\
(n=238)\end{array}$ & $p$-Value & $\beta$-Coefficient & OR $(95 \% \mathrm{CI})$ \\
\hline Age & - & $62.39( \pm 11.02)$ & $63.53( \pm 10.54)$ & NS & 0.01 & $1.00(1.00-1.02)$ \\
\hline \multirow[t]{2}{*}{ Sex } & Male & $420(53.37)$ & $126(52.94)$ & - & - & - \\
\hline & Female & $367(46.63)$ & $112(47.06)$ & NS & 0.02 & $1.02(0.76-1.36)$ \\
\hline \multirow[t]{5}{*}{ Ethnicity } & Non-Hispanic white & $369(46.88)$ & $91(38.23)$ & - & - & - \\
\hline & Non-Hispanic black & $166(21.09)$ & $74(31.09)$ & $* *$ & 0.59 & $1.81(1.27-2.58)$ \\
\hline & Mexican-American & $138(17.54)$ & $41(17.23)$ & NS & 0.19 & $1.21(0.79-1.83)$ \\
\hline & Other Hispanic & $89(11.31)$ & $28(11.77)$ & NS & 0.24 & $1.28(0.79-2.07)$ \\
\hline & Other race-including multiracial & $25(3.18)$ & $4(1.68)$ & NS & -0.43 & $0.65(0.22-1.91)$ \\
\hline \multirow[t]{5}{*}{ Education } & Less than 9th grade & $148(18.8)$ & $54(22.69)$ & - & - & - \\
\hline & 9-11th grade ${ }^{ \pm}$ & $148(18.8)$ & $51(21.43)$ & NS & -0.06 & $0.94(0.61-1.47)$ \\
\hline & High school graduate/GED or equivalent & $199(25.29)$ & $56(23.53)$ & NS & -0.26 & $0.77(0.5-1.19)$ \\
\hline & Some college or AA degree & $172(21.86)$ & $55(23.11)$ & NS & -0.13 & $0.88(0.57-1.35)$ \\
\hline & College graduate or above & $120(15.25)$ & $22(9.24)$ & * & -0.69 & $0.50 .29-0.87)$ \\
\hline \multirow[t]{6}{*}{ Marital status } & Married & $464(58.96)$ & $133(55.88)$ & - & - & - \\
\hline & Widowed & $119(15.12)$ & $36(15.13)$ & NS & 0.05 & $1.06(0.69-1.61)$ \\
\hline & Divorced & $90(11.44)$ & $37(15.55)$ & NS & 0.36 & $1.43(0.94-2.2)$ \\
\hline & Separated & $32(4.07)$ & $7(2.94)$ & NS & -0.27 & $0.76(0.33-1.77)$ \\
\hline & Never married & $53(6.73)$ & $18(7.56)$ & NS & 0.17 & $1.19(0.67-2.09)$ \\
\hline & Living with partner & $29(3.68)$ & $7(2.94)$ & NS & -0.17 & $0.84(0.36-1.97)$ \\
\hline \multirow[t]{3}{*}{ Family income } & $\$ 0-\$ 4999$ & $11(1.4)$ & $3(1.26)$ & - & - & - \\
\hline & $\$ 5000-\$ 9999$ & $47(5.97)$ & $12(5.04)$ & NS & -0.07 & $0.94(0.23-3.89)$ \\
\hline & $\$ 10,000-\$ 14,999$ & $82(10.42)$ & $22(9.25)$ & NS & -0.02 & $0.98(0.25-3.84)$ \\
\hline
\end{tabular}


Table 1. Cont.

\begin{tabular}{|c|c|c|c|c|c|c|}
\hline $\begin{array}{c}\text { Characteristics } \\
\text { Mean }( \pm \mathrm{SD}) \\
n(\%)\end{array}$ & & $\begin{array}{c}\text { Diabetes with } \\
\text { Non-Diabetic } \\
\text { Retinopathy } \\
(n=787)\end{array}$ & $\begin{array}{c}\text { Diabetic } \\
\text { Retinopathy } \\
(n=238)\end{array}$ & $p$-Value & $\beta$-Coefficient & OR $(95 \% \mathrm{CI})$ \\
\hline & $\$ 15,000-\$ 19,999$ & $68(8.64)$ & $22(9.25)$ & NS & 0.17 & $1.19(0.30-4.64)$ \\
\hline & $\$ 20,000-\$ 24,999$ & 69 (8.77) & $25(10.5)$ & NS & 0.28 & $1.33(0.34-5.16)$ \\
\hline & $\$ 25,000-\$ 34,999$ & $103(13.09)$ & $42(17.65)$ & NS & 0.40 & $1.50(0.40-5.63)$ \\
\hline & $\$ 35,000-\$ 44,999$ & $68(8.64)$ & $23(9.66)$ & NS & 0.22 & $1.24(0.32-4.84)$ \\
\hline & $\$ 45,000-\$ 54,999$ & 55 (6.99) & $12(5.04)$ & NS & -0.22 & $0.80(0.19-3.31)$ \\
\hline & $\$ 55,000-\$ 64,999$ & $41(5.21)$ & $15(6.3)$ & NS & 0.29 & $1.34(0.33-5.48)$ \\
\hline & $\$ 65,000-\$ 74,999$ & $36(4.57)$ & $6(2.52)$ & NS & -0.49 & $0.61(0.13-2.86)$ \\
\hline & $\$ 75,000-\$ 99,999$ & 44 (5.59) & $12(5.04)$ & NS & 0.00 & $1.00(0.24-4.17)$ \\
\hline & $\geq \$ 100,000$ & $88(11.18)$ & $17(7.14)$ & NS & -0.35 & $0.71(0.18-2.81)$ \\
\hline & Over $\$ 20,000$ & $31(3.94)$ & $9(3.78)$ & NS & 0.06 & $1.06(0.24-4.66)$ \\
\hline & Under $\$ 20,000$ & $17(2.16)$ & $2(0.84)$ & NS & -0.84 & $0.43(0.06-3.01)$ \\
\hline & Missing & 27 (3.43) & $16(6.73)$ & - & - & - \\
\hline Diabetes duration & - & $9.05( \pm 11.05)$ & $16.33( \pm 12.57)$ & $* * *$ & 0.05 & $1.05(1.04-1.07)$ \\
\hline
\end{tabular}

Notes: The NHANES codes used in our selection criteria of people with diabetes include: 1, self-reported diabetes (DIQ010); 2, on anti-diabetes drugs (DIQ070); 3, taking insulin (DIQ050); 4, fasting blood sugar (FBS) $\geq 6.1$ (110 mg/dL) (LBDGLUSI); 5, random blood sugar (RBS) $\geq 11.1$ (200 mg/dL) (LBDSGLSI); 6 , oral glucose tolerance test (OGTT) $\geq 200 \mathrm{mg} / \mathrm{dL}$ (LBDGLTSI); 7, glycohemoglobin $(\mathrm{HbA1c}) \geq 6.5 \%$ (LBXGH). Ethnicity, education, and diabetes duration contain at least one term that is statistically significant. \pm includes 12 th grade with no diploma. NS, not statistically significant; ${ }^{*}, p<0.05 ;{ }^{* *}, p<0.01 ; * *, p<0.001$. 
Table 2. Retinal lesions that constitute diabetic retinopathy outcome.

\begin{tabular}{|c|c|c|c|c|c|}
\hline Clinical Signs of DR & $n(\%)$ & $\beta$-Coefficient & OR $(95 \%$ CI $)$ & $p$-Value & FDR-Adjusted $p$-Value \\
\hline Retinal microaneurysms only, worse eye & $129(12.59)$ & 5.67 & $288.87(127.66-653.68)$ & $* * *$ & $* * *$ \\
\hline Retinal hard exudate, worse eye & $86(8.39)$ & 3.72 & $41.34(20.31-84.17)$ & $* * *$ & $* * *$ \\
\hline Retinal blot haemorrhages, worse eye & $47(4.59)$ & 3.36 & $28.71(14.11-58.42)$ & $* * *$ & $* * *$ \\
\hline Retinal soft exudate, worse eye & $76(7.41)$ & 4.2 & $66.49(26.44-167.21)$ & $* * *$ & $* * *$ \\
\hline IRMA, worse eye & $62(6.05)$ & 2.85 & $17.36(9.06-33.26)$ & $* * *$ & $* * *$ \\
\hline Macular oedema, worse eye & $51(4.98)$ & 3.88 & $48.24(17.18-135.44)$ & $* * *$ & $* * *$ \\
\hline Retinal fibrous proliferation, worse eye & $19(1.85)$ & 3.41 & $30.19(6.92-131.67)$ & $* * *$ & $* * *$ \\
\hline Macular oedema in centre, worse eye & $26(2.54)$ & 4.53 & $92.33(12.45-684.9)$ & $* * *$ & $* * *$ \\
\hline Retinal new vessels elsewhere, worse eye & $15(1.46)$ & 3.12 & $22.68(5.08-101.23)$ & $* * *$ & $* * *$ \\
\hline
\end{tabular}

Notes: lesions are taken from the NHANES ophthalmology—retinal imaging (OPXRET_E) dataset. Only lesions with false discovery rate (FDR)-adjusted $p \leq 0.05$ are listed. Soft exudate is now termed cotton wool spots. NS, not statistically significant; IRMA, intraretinal microvascular abnormalities; ${ }^{* * *}, p<0.001$.

Table 3. Laboratory variables associated with retinopathy in individuals with diabetes.

\begin{tabular}{|c|c|c|c|c|c|c|c|c|c|c|c|c|c|c|c|c|}
\hline \multirow[b]{2}{*}{ Description } & \multicolumn{4}{|c|}{ Unadjusted/Non-Covariate Adjusted } & \multicolumn{4}{|c|}{ Age-Adjusted } & \multicolumn{4}{|c|}{ Ethnicity-Adjusted } & \multicolumn{4}{|c|}{ Diabetes Duration-Adjusted } \\
\hline & $\beta$-Coefficient & OR $(95 \% \mathrm{CI})$ & $p$-Value & $\begin{array}{c}\text { FDR-Adjusted } \\
p \text {-Value }\end{array}$ & $\beta$-Coefficient & OR $(95 \% \mathrm{CI})$ & $p$-Value & $\begin{array}{l}\text { FDR-Adjusted } \\
p \text {-Value }\end{array}$ & $\beta$-Coefficient & OR $(95 \% \mathrm{CI})$ & $p$-Value & $\begin{array}{l}\text { FDR-Adjusted } \\
p \text {-Value }\end{array}$ & $\beta$-Coefficient & OR $(95 \% \mathrm{CI})$ & $p$-Value & $\begin{array}{c}\text { FDR-Adjusted } \\
p \text {-Value }\end{array}$ \\
\hline $\begin{array}{l}\text { Glycohemoglobin } \\
(\mathrm{HbA1c})(\%)\end{array}$ & 0.82 & $\begin{array}{c}2.27 \\
(1.84-2.8) \\
\end{array}$ & $* * *$ & $* * *$ & 0.85 & $\begin{array}{l}2.34 \\
(1.87-2.92)\end{array}$ & $* * *$ & $* *$ & 0.83 & $\begin{array}{c}2.28 \\
(1.82-2.87) \\
\end{array}$ & $* * *$ & $* *$ & 0.72 & $\begin{array}{l}2.05 \\
(1.55-2.73)\end{array}$ & $* * *$ & * \\
\hline $\begin{array}{c}\text { Random Glucose, } \\
\text { serum } \\
\text { (RBS) (mmol/L) }\end{array}$ & 0.54 & $\begin{array}{c}1.72 \\
(1.42-2.08)\end{array}$ & $* * *$ & ** & 0.57 & $\begin{array}{c}1.77 \\
(1.45-2.15)\end{array}$ & *** & * & 0.54 & $\begin{array}{c}1.71 \\
(1.41-2.09)\end{array}$ & *** & * & 0.36 & $\begin{array}{c}1.43 \\
(1.1-1.86)\end{array}$ & * & NS \\
\hline $\begin{array}{l}\text { Osmolality } \\
(\mathrm{mmol} / \mathrm{Kg})\end{array}$ & 0.49 & $\begin{array}{c}1.63 \\
(1.34-1.99) \\
\end{array}$ & $* * *$ & * & 0.45 & $\begin{array}{c}1.57 \\
(1.3-1.9) \\
\end{array}$ & $* * *$ & * & 0.49 & $\begin{array}{c}1.63 \\
(1.34-1.99) \\
\end{array}$ & $* * *$ & NS & 0.39 & $\begin{array}{c}1.48 \\
(1.12-1.94) \\
\end{array}$ & * & * \\
\hline $\begin{array}{l}\text { Albumin, urine } \\
\quad(\mathrm{mg} / \mathrm{L})\end{array}$ & 0.45 & $\begin{array}{c}1.57 \\
(1.28-1.93) \\
\end{array}$ & $* * *$ & * & 0.43 & $\begin{array}{c}1.53 \\
(1.24-1.88) \\
\end{array}$ & ** & * & 0.42 & $\begin{array}{c}1.53 \\
(1.25-1.87) \\
\end{array}$ & $* *$ & NS & 0.25 & $\begin{array}{c}1.28 \\
(0.98-1.68) \\
\end{array}$ & NS & NS \\
\hline Haemoglobin (g/dL) & -0.33 & $\begin{array}{c}0.72 \\
(0.61-0.85) \\
\end{array}$ & ** & * & -0.30 & $\begin{array}{c}0.74 \\
(0.63-0.88) \\
\end{array}$ & ** & * & -0.29 & $\begin{array}{c}0.75 \\
(0.62-0.9) \\
\end{array}$ & ** & NS & 0.00 & $\begin{array}{c}1 \\
(0.73-1.37) \\
\end{array}$ & NS & NS \\
\hline $\begin{array}{l}\text { Fasting Glucose(FBS) } \\
\text { (mmol/L) }\end{array}$ & 0.49 & $\begin{array}{c}1.63 \\
(1.28-2.07)\end{array}$ & ** & * & 0.51 & $\begin{array}{c}1.66 \\
(1.3-2.13)\end{array}$ & ** & * & 0.46 & $\begin{array}{c}1.59 \\
(1.25-2.02)\end{array}$ & ** & NS & 0.22 & $\begin{array}{c}1.24 \\
(0.93-1.66)\end{array}$ & NS & NS \\
\hline $\begin{array}{l}\text { 4-(methylnitrosamino)- } \\
\text { 1-(3-pyridyl)-1-butanol } \\
\text { (NNAL), urine } \\
\text { (ng/mL) }\end{array}$ & -0.26 & $\begin{array}{c}0.77 \\
(0.67-0.88)\end{array}$ & ** & NS & -0.20 & $\begin{array}{c}0.82 \\
(0.71-0.94)\end{array}$ & * & NS & -0.28 & $\begin{array}{c}0.75 \\
(0.65-0.87)\end{array}$ & ** & NS & -0.25 & $\begin{array}{c}0.78 \\
(0.52-1.19)\end{array}$ & NS & NS \\
\hline Iodine, urine (ug/L) & -0.17 & $\begin{array}{c}0.84 \\
(0.76-0.93)\end{array}$ & ** & NS & -0.21 & $\begin{array}{c}0.81 \\
(0.73-0.9)\end{array}$ & ** & * & -0.15 & $\begin{array}{c}0.86 \\
(0.77-0.96)\end{array}$ & * & NS & -0.29 & $\begin{array}{c}0.75 \\
(0.63-0.89)\end{array}$ & $* *$ & * \\
\hline
\end{tabular}


Table 3. Cont

\begin{tabular}{|c|c|c|c|c|c|c|c|c|c|c|c|c|c|c|c|c|}
\hline \multirow[b]{2}{*}{ Description } & \multicolumn{4}{|c|}{ Unadjusted/Non-Covariate Adjusted } & \multicolumn{4}{|c|}{ Age-Adjusted } & \multicolumn{4}{|c|}{ Ethnicity-Adjusted } & \multicolumn{4}{|c|}{ Diabetes Duration-Adjusted } \\
\hline & $\beta$-Coefficient & OR $(95 \% \mathrm{CI})$ & $p$-Value & $\begin{array}{c}\text { FDR-Adjusted } \\
p \text {-Value }\end{array}$ & $\beta$-Coefficient & OR $(95 \% \mathrm{CI})$ & $p$-Value & $\begin{array}{c}\text { FDR-Adjusted } \\
p \text {-Value }\end{array}$ & $\beta$-Coefficient & OR $(95 \%$ CI) & $p$-Value & $\begin{array}{l}\text { FDR-Adjusted } \\
p \text {-Value }\end{array}$ & $\beta$-Coefficient & OR $(95 \% \mathrm{CI})$ & $p$-Value & $\begin{array}{c}\text { FDR-Adjusted } \\
p \text {-Value }\end{array}$ \\
\hline Cobalt, urine (ug/L) & -0.51 & $\begin{array}{c}0.6 \\
(0.44-0.82)\end{array}$ & $* *$ & NS & -0.50 & $\begin{array}{c}0.6 \\
(0.45-0.82)\end{array}$ & ** & * & -0.51 & $\begin{array}{c}0.6 \\
(0.44-0.81)\end{array}$ & $* *$ & NS & -0.52 & $\begin{array}{c}0.59 \\
(0.45-0.78)\end{array}$ & ** & * \\
\hline Haematocrit $(\%)$ & -0.31 & $\begin{array}{c}0.73 \\
(0.6-0.89) \\
\end{array}$ & $* *$ & NS & -0.28 & $\begin{array}{c}0.76 \\
(0.62-0.92)\end{array}$ & * & NS & -0.28 & $\begin{array}{c}0.75 \\
(0.62-0.92)\end{array}$ & * & NS & -0.03 & $\begin{array}{c}0.97 \\
(0.7-1.35) \\
\end{array}$ & NS & NS \\
\hline $\begin{array}{l}\text { Blood urea nitrogen } \\
\text { (BUN) (mmol/L) }\end{array}$ & 0.33 & $\begin{array}{c}1.4 \\
(1.13-1.73)\end{array}$ & $* *$ & NS & 0.27 & $\begin{array}{c}1.31 \\
(1.01-1.71)\end{array}$ & NS & NS & 0.35 & $\begin{array}{c}1.43 \\
(1.16-1.75)\end{array}$ & ** & NS & 0.15 & $\begin{array}{c}1.17 \\
(0.87-1.57)\end{array}$ & NS & NS \\
\hline Albumin $(g / L)$ & -0.22 & $\begin{array}{c}0.8 \\
(0.69-0.93)\end{array}$ & * & NS & -0.21 & $\begin{array}{c}0.81 \\
(0.69-0.94)\end{array}$ & * & NS & -0.19 & $\begin{array}{c}0.83 \\
(0.7-0.99)\end{array}$ & NS & NS & -0.09 & $\begin{array}{c}0.92 \\
(0.74-1.14)\end{array}$ & NS & NS \\
\hline $\begin{array}{c}\text { Urinary Triclosan } \\
(\mathrm{ng} / \mathrm{mL})\end{array}$ & -0.42 & $\begin{array}{c}0.65 \\
(0.49-0.88) \\
\end{array}$ & * & NS & -0.40 & $\begin{array}{c}0.67 \\
(0.5-0.89) \\
\end{array}$ & * & NS & -0.42 & $\begin{array}{c}0.66 \\
(0.49-0.89) \\
\end{array}$ & * & NS & -0.60 & $\begin{array}{c}0.55 \\
(0.36-0.83) \\
\end{array}$ & * & * \\
\hline $\begin{array}{c}\text { Mean cell } \\
\text { haemoglobin }(\mathrm{pg})\end{array}$ & -0.24 & $\begin{array}{c}0.79 \\
(0.66-0.93) \\
\end{array}$ & * & NS & -0.27 & $\begin{array}{c}0.77 \\
(0.65-0.91) \\
\end{array}$ & ** & * & -0.17 & $\begin{array}{c}0.84 \\
(0.7-1.02) \\
\end{array}$ & NS & NS & -0.01 & $\begin{array}{c}0.99 \\
(0.76-1.28) \\
\end{array}$ & NS & NS \\
\hline Lead, urine $(\mu \mathrm{g} / \mathrm{L})$ & -0.40 & $\begin{array}{c}0.67 \\
(0.49-0.91) \\
\end{array}$ & * & NS & -0.40 & $\begin{array}{c}0.67 \\
(0.49-0.91) \\
\end{array}$ & * & NS & -0.41 & $\begin{array}{c}0.66 \\
(0.49-0.9) \\
\end{array}$ & * & NS & -0.43 & $\begin{array}{c}0.65 \\
(0.43-0.99) \\
\end{array}$ & NS & NS \\
\hline $\begin{array}{l}\text { Creatinine, urine } \\
(\mu \mathrm{mol} / \mathrm{L})\end{array}$ & -0.22 & $\begin{array}{c}0.8 \\
(0.67-0.97)\end{array}$ & * & NS & -0.19 & $\begin{array}{c}0.83 \\
(0.68-1)\end{array}$ & NS & NS & -0.27 & $\begin{array}{c}0.77 \\
(0.64-0.92)\end{array}$ & * & NS & -0.28 & $\begin{array}{c}0.76 \\
(0.64-0.9) \\
\end{array}$ & ** & * \\
\hline $\begin{array}{c}\text { Alanine } \\
\text { aminotransferase } \\
\text { (ALT) (U/L) }\end{array}$ & -0.25 & $\begin{array}{c}0.78 \\
(0.62-0.97)\end{array}$ & * & NS & -0.20 & $\begin{array}{c}0.82 \\
(0.66-1.02)\end{array}$ & NS & NS & -0.22 & $\begin{array}{c}0.81 \\
(0.64-1.01)\end{array}$ & NS & NS & -0.09 & $\begin{array}{c}0.92 \\
(0.68-1.23)\end{array}$ & NS & NS \\
\hline Creatinine $(\mu \mathrm{mol} / \mathrm{L})$ & 0.24 & $\begin{array}{c}1.27 \\
(1.04-1.54) \\
\end{array}$ & * & NS & 0.18 & $\begin{array}{c}1.19 \\
(0.96-1.48) \\
\end{array}$ & NS & NS & 0.20 & $\begin{array}{c}1.23 \\
(0.99-1.53) \\
\end{array}$ & NS & NS & 0.13 & $\begin{array}{c}1.14 \\
(0.89-1.45) \\
\end{array}$ & NS & NS \\
\hline $\begin{array}{l}\text { Red blood cell count } \\
\text { (million cells } / \mu \mathrm{L})\end{array}$ & -0.19 & $\begin{array}{c}0.82 \\
(0.7-0.97) \\
\end{array}$ & * & NS & -0.13 & $\begin{array}{c}0.88 \\
(0.74-1.04)\end{array}$ & NS & NS & -0.19 & $\begin{array}{c}0.83 \\
(0.7-0.98) \\
\end{array}$ & NS & NS & 0.01 & $\begin{array}{c}1.01 \\
(0.74-1.37)\end{array}$ & NS & NS \\
\hline $\begin{array}{l}\text { Mean cell volume } \\
(\mathrm{fL})\end{array}$ & -0.21 & $\begin{array}{c}0.81 \\
(0.68-0.98)\end{array}$ & * & NS & -0.25 & $\begin{array}{c}0.78 \\
(0.65-0.93)\end{array}$ & * & NS & -0.15 & $\begin{array}{c}0.86 \\
(0.71-1.06)\end{array}$ & NS & NS & -0.05 & $\begin{array}{c}0.95 \\
(0.72-1.26)\end{array}$ & NS & NS \\
\hline $\begin{array}{l}\text { Platelet count } \\
(1000 \text { cells } / \mu \mathrm{L})\end{array}$ & -0.21 & $\begin{array}{c}0.81 \\
(0.68-0.98)\end{array}$ & * & NS & -0.18 & $\begin{array}{c}0.83 \\
(0.68-1.02) \\
\end{array}$ & NS & NS & -0.23 & $\begin{array}{c}0.79 \\
(0.67-0.94)\end{array}$ & * & NS & -0.29 & $\begin{array}{c}0.75 \\
(0.57-0.99)\end{array}$ & NS & NS \\
\hline $\begin{array}{l}\text { Mean platelet } \\
\text { volume }(\mathrm{fL})\end{array}$ & 0.24 & $\begin{array}{c}1.27 \\
(1.04-1.55)\end{array}$ & * & NS & 0.26 & $\begin{array}{c}1.3 \\
(1.06-1.59)\end{array}$ & * & NS & 0.22 & $\begin{array}{c}1.25 \\
(1.02-1.53)\end{array}$ & NS & NS & 0.09 & $\begin{array}{c}1.09 \\
(0.83-1.43)\end{array}$ & NS & NS \\
\hline Cotinine $(\mathrm{ng} / \mathrm{mL})$ & -0.15 & $\begin{array}{c}0.86 \\
(0.76-0.99) \\
\end{array}$ & * & NS & -0.09 & $\begin{array}{c}0.91 \\
(0.79-1.04) \\
\end{array}$ & NS & NS & -0.18 & $\begin{array}{c}0.84 \\
(0.74-0.95) \\
\end{array}$ & * & NS & -0.06 & $\begin{array}{c}0.94 \\
(0.66-1.34) \\
\end{array}$ & NS & NS \\
\hline Insulin (pmo//L) & -0.32 & $\begin{array}{c}0.73 \\
(0.55-0.97)\end{array}$ & * & NS & -0.30 & $\begin{array}{c}0.74 \\
(0.55-1)\end{array}$ & NS & NS & -0.29 & $\begin{array}{c}0.75 \\
(0.56-1) \\
\end{array}$ & NS & NS & -0.22 & $\begin{array}{c}0.8 \\
(0.51-1.26)\end{array}$ & NS & NS \\
\hline $\begin{array}{c}\text { Blood cadmium } \\
\text { (nmol/L) }\end{array}$ & -0.23 & $\begin{array}{c}0.8 \\
(0.65-0.97)\end{array}$ & * & NS & -0.25 & $\begin{array}{c}0.78 \\
(0.64-0.95)\end{array}$ & * & NS & -0.23 & $\begin{array}{c}0.79 \\
(0.64-0.98)\end{array}$ & NS & NS & -0.28 & $\begin{array}{c}0.76 \\
(0.5-1.16)\end{array}$ & NS & NS \\
\hline $\begin{array}{l}\text { Urinary perchlorate } \\
(\mathrm{ng} / \mathrm{mL})\end{array}$ & -0.25 & $\begin{array}{c}0.78 \\
(0.63-0.95)\end{array}$ & * & NS & -0.24 & $\begin{array}{c}0.78 \\
(0.63-0.98)\end{array}$ & * & NS & -0.24 & $\begin{array}{c}0.78 \\
(0.64-0.96)\end{array}$ & * & NS & -0.31 & $\begin{array}{c}0.73 \\
(0.53-1.01)\end{array}$ & NS & NS \\
\hline $\begin{array}{c}\text { Urinary nitrate } \\
(\mathrm{ng} / \mathrm{mL})\end{array}$ & -0.28 & $\begin{array}{c}0.75 \\
(0.6-0.94)\end{array}$ & * & NS & -0.23 & $\begin{array}{c}0.79 \\
(0.63-1)\end{array}$ & NS & NS & -0.27 & $\begin{array}{c}0.76 \\
(0.6-0.96)\end{array}$ & * & NS & -0.34 & $\begin{array}{c}0.71 \\
(0.55-0.91)\end{array}$ & * & NS \\
\hline Cesium, urine $(\mu \mathrm{g} / \mathrm{L})$ & -0.33 & $\begin{array}{c}0.72 \\
(0.54-0.95)\end{array}$ & * & NS & -0.32 & $\begin{array}{c}0.72 \\
(0.55-0.96)\end{array}$ & * & NS & -0.33 & $\begin{array}{c}0.72 \\
(0.54-0.95)\end{array}$ & * & NS & -0.34 & $\begin{array}{c}0.71 \\
(0.47-1.06)\end{array}$ & NS & NS \\
\hline $\begin{array}{l}\text { Thallium, urine } \\
(\mu \mathrm{g} / \mathrm{L})\end{array}$ & -0.40 & $\begin{array}{c}0.67 \\
(0.48-0.95)\end{array}$ & * & NS & -0.39 & $\begin{array}{c}0.68 \\
(0.48-0.96)\end{array}$ & * & NS & -0.42 & $\begin{array}{c}0.66 \\
(0.47-0.92)\end{array}$ & * & NS & -0.48 & $\begin{array}{c}0.62 \\
(0.4-0.94)\end{array}$ & * & NS \\
\hline
\end{tabular}


Table 3. Cont.

\begin{tabular}{|c|c|c|c|c|c|c|c|c|c|c|c|c|c|c|c|c|}
\hline \multirow[b]{2}{*}{ Description } & \multicolumn{4}{|c|}{ Unadjusted/Non-Covariate Adjusted } & \multicolumn{4}{|c|}{ Age-Adjusted } & \multicolumn{4}{|c|}{ Ethnicity-Adjusted } & \multicolumn{4}{|c|}{ Diabetes Duration-Adjusted } \\
\hline & $\beta$-Coefficient & OR $(95 \% \mathrm{CI})$ & $p$-Value & $\begin{array}{c}\text { FDR-Adjusted } \\
p \text {-Value }\end{array}$ & $\beta$-Coefficient & OR $(95 \% \mathrm{CI})$ & $p$-Value & $\begin{array}{c}\text { FDR-Adjusted } \\
p \text {-Value }\end{array}$ & $\beta$-Coefficient & OR $(95 \% \mathrm{CI})$ & $p$-Value & $\begin{array}{c}\text { FDR-Adjusted } \\
p \text {-Value }\end{array}$ & $\beta$-Coefficient & OR $(95 \% \mathrm{CI})$ & $p$-Value & $\begin{array}{c}\text { FDR-Adjusted } \\
p \text {-Value }\end{array}$ \\
\hline $\begin{array}{c}\text { Vitamin D2 or D3 } \\
\text { 25OHD2 + 25OHD3 } \\
\text { (nmol/L) }\end{array}$ & -0.24 & $\begin{array}{c}0.79 \\
(0.65-0.95)\end{array}$ & * & NS & -0.25 & $\begin{array}{c}0.78 \\
(0.65-0.94)\end{array}$ & * & NS & -0.18 & $\begin{array}{c}0.83 \\
(0.66-1.04)\end{array}$ & NS & NS & -0.20 & $\begin{array}{c}0.82 \\
(0.61-1.1)\end{array}$ & NS & NS \\
\hline $\begin{array}{c}\text { Blood Toluene } \\
\text { (ng/mL) }\end{array}$ & -0.21 & $\begin{array}{c}0.81 \\
(0.68-0.96) \\
\end{array}$ & * & NS & -0.20 & $\begin{array}{c}0.82 \\
(0.69-0.97) \\
\end{array}$ & * & NS & -0.22 & $\begin{array}{c}0.8 \\
(0.65-0.98) \\
\end{array}$ & NS & NS & -0.30 & $\begin{array}{c}0.74 \\
(0.45-1.21) \\
\end{array}$ & NS & NS \\
\hline $\begin{array}{c}\text { C-reactive protein } \\
(\mathrm{m} / \mathrm{dL})\end{array}$ & -0.19 & 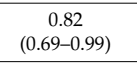 & NS & NS & -0.18 & $\begin{array}{c}0.83 \\
(0.69-1.01) \\
\end{array}$ & NS & NS & -0.25 & $\begin{array}{c}0.78 \\
(0.66-0.92) \\
\end{array}$ & * & NS & -0.27 & 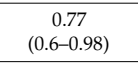 & * & NS \\
\hline Barium, urine $(\mu g / \mathrm{L})$ & -0.39 & $\begin{array}{c}0.68 \\
(0.47-0.99) \\
\end{array}$ & NS & NS & -0.38 & $\begin{array}{c}0.69 \\
(0.48-0.99) \\
\end{array}$ & NS & NS & -0.38 & $\begin{array}{c}0.68 \\
(0.47-1)\end{array}$ & NS & NS & -0.44 & $\begin{array}{c}0.64 \\
(0.47-0.87) \\
\end{array}$ & * & * \\
\hline $\begin{array}{c}\text { Urinary } \\
\text { 4-tert-octylphenol } \\
\text { (ng/mL) }\end{array}$ & -0.34 & $\begin{array}{c}0.71 \\
(0.45-1.12)\end{array}$ & NS & NS & -0.33 & $\begin{array}{c}0.72 \\
(0.46-1.12)\end{array}$ & NS & NS & -0.42 & $\begin{array}{c}0.66 \\
(0.43-1.01)\end{array}$ & NS & NS & -0.82 & $\begin{array}{c}0.44 \\
(0.23-0.87)\end{array}$ & * & NS \\
\hline $\begin{array}{l}\text { Dimethyl } \\
\text { dithiophosphate } \\
(\mu \mathrm{g} / \mathrm{L})\end{array}$ & -0.31 & $\begin{array}{c}0.74 \\
(0.51-1.06)\end{array}$ & NS & NS & -0.35 & $\begin{array}{c}0.71 \\
(0.49-1.01)\end{array}$ & NS & NS & -0.21 & $\begin{array}{c}0.81 \\
(0.59-1.12)\end{array}$ & NS & NS & -0.67 & $\begin{array}{c}0.51 \\
(0.29-0.91)\end{array}$ & * & NS \\
\hline
\end{tabular}

Variables were first tested in an unadjusted/non-covariate adjusted analysis, and then again adjusting for age, ethnicity, and diabetes duration. To provide a broad overview, any variable passing nominal (i.e., prior to FDR-correction) $p \leq 0.05$ from either the non-covariate adjusted or any of the covariate-adjusted analyses are listed. OR, odds ratio; NS, not statistically significant; ${ }^{*}, p<0.05 ; * *, p<0.01$; ${ }^{* * *}, p<0.001$.

Table 4. RandomForest ${ }^{\mathrm{TM}}$-selected variables (features) ranked by mean decrease accuracy.

\begin{tabular}{cccc}
\hline Marker & Group & Mean Decrease Accuracy & Mean Decrease Gini \\
\hline Glycohemoglobin $(\%)$ & Diabetes status & 31.93719324 & 21.75225602 \\
\hline C-reactive protein $(\mathrm{mg} / \mathrm{dL})$ & Immune markers & 11.51047187 & 10.1975135 \\
\hline Potassium $(\mathrm{mmol} / \mathrm{L})$ & Renal function & 11.44142126 & 8.198116194 \\
\hline Albumin, urine $(\mathrm{mg} / \mathrm{L})$ & Renal function & 8.220187056 & 10.21346621 \\
\hline Monocyte number $(1000$ cells/uL) & Immune markers & 7.663589246 & 3.957711466 \\
\hline Osmolality $(\mathrm{mmol} / \mathrm{Kg})$ & Renal function & 7.510989556 & 5.33445026 \\
\hline White blood cell count $(1000$ cells/uL) & Immune markers & 7.440157644 & 4.405168072 \\
\hline Blood urea nitrogen $(\mathrm{mmol} / \mathrm{L})$ & Renal function & 7.224789174 & 5.073947519 \\
\hline Segmented neutrophils num $(1000$ cell//uL) & Immune markers & 7.020397225 & 4.067402271 \\
\hline Fasting Glucose $(\mathrm{mmol} / \mathrm{L})$ & Diabetes status & 6.563694988 & 2.826827797 \\
\hline Red cell distribution width $(\%)$ & Haematocrit & 6.138538515 & 4.947185792 \\
\hline
\end{tabular}


Table 4. Cont.

\begin{tabular}{|c|c|c|c|}
\hline Marker & Group & Mean Decrease Accuracy & Mean Decrease Gini \\
\hline Urinary nitrate $(\mathrm{ng} / \mathrm{mL})$ & Renal function & 5.899174386 & 5.844258103 \\
\hline Glucose, serum (mmol/L) & Diabetes status & 5.85339684 & 4.77357191 \\
\hline 2-hydroxyphenanthrene (ng/L) & Toxins/Metals & 4.028082549 & 2.307374348 \\
\hline $\mathrm{MCHC}(\mathrm{g} / \mathrm{dL})$ & Haematocrit & 3.936015913 & 3.896804592 \\
\hline Creatinine $(\mu \mathrm{mol} / \mathrm{L})$ & Renal function & 3.530916132 & 3.747500758 \\
\hline Mono-2-ethyl-5-carboxypentyl phthalate & Toxins/Metals & 2.996581682 & 2.212151134 \\
\hline Blood Nitromethane (pg/mL) & Toxins/Metals & 2.936160917 & 3.31634662 \\
\hline Phosphorus (mmol/L) & Toxins/Metals & 2.819084205 & 3.768671211 \\
\hline Total Cholesterol (mmol/L) & Sterols & 2.448578413 & 3.833301666 \\
\hline Enterodiol (ng/mL) & Sterols & 2.401651721 & 2.722762228 \\
\hline Haematocrit $(\%)$ & Haematocrit & 2.364741874 & 4.841281382 \\
\hline Mono-n-octyl phthalate (ng/mL) & Toxins/Metals & 2.215508752 & 0.231647322 \\
\hline Mean cell haemoglobin (pg) & Haematocrit & 2.183482824 & 4.292282119 \\
\hline Gamma glutamyl transferase (U/L) & Liver Function & 1.989695745 & 4.015070221 \\
\hline Systolic blood pressure & Blood pressure & 1.892815461 & 8.85430752 \\
\hline Blood o-Xylene (ng/mL) & Toxins/Metals & 1.670964308 & 3.708248225 \\
\hline Lactate dehydrogenase LDH (U/L) & Liver Function & 1.593869272 & 4.231659273 \\
\hline 9-hydroxyfluorene (ng/L) & Toxins/Metals & 1.430814333 & 2.206778568 \\
\hline Cholesterol (mmol/L) & Sterols & 1.201366974 & 4.025207689 \\
\hline 3-hydroxyphenanthrene (ng/L) & Toxins/Metals & 1.00569817 & 1.63630888 \\
\hline
\end{tabular}

Notes: The model was initially trained on all laboratory variables in an unsupervised fashion, with Kappa-based model tuning to select the optimum values for 'mtry' (the ideal number of variables to randomly sample) and 'ntrees' (the ideal number of trees). Only variables contributing $>1 \%$ mean decrease in accuracy from the initial model were retained, followed by recursive steps to remove low-informative variables. Variables are manually assigned to groups based on similar organ function or other characteristic. Mean decrease accuracy is the estimated decrease in overall model accuracy once a given parameter is removed from the model, and therefore serves as a useful metric for ordering importance. The Gini importance measure relates to the 'splitting' criterion that is employed in classification trees, and it is known to be less biased for continuous variables (26), which naturally have more splitting points compared to categorical variables. 'Group' is manually curated. 


\subsection{Replication Cohort}

In the NHANES 2005-2006 replication cohort, we performed penalised regression and RandomForest ${ }^{\mathrm{TM}}$ in the same way as per the 2007-2008 cohort. Our penalised regression model identified urinary albumin (mg/L), cockroach $\mathrm{IgE}$ antibody (kU/L), HbA1c (\%), haemoglobin $(\mathrm{g} / \mathrm{dL})$, and urinary nitrate $(\mathrm{ng} / \mathrm{mL})$, with a model accuracy of $73.16 \%$ and AUC 0.76 (95\% CI: 0.73-0.78). RandomForest ${ }^{\mathrm{TM}}$ identified $\mathrm{HbA1c}(\%)$ as the variable contributing most to accuracy (mean decrease $16.96 \%$ ), with many other variables contributing appreciable accuracy to the overall model (Supplementary Table S3)—the overall model accuracy was 72.98\% and AUC 0.68 (95\% CI: 0.61-0.75).

\subsection{Final Clinical Risk Models}

The 31 features identified by RandomForest ${ }^{\mathrm{TM}}$ (Table 4) were grouped into different categories of blood tests, according to diabetes status, haematocrit values, blood pressure (BP), immune markers, renal function, sterols, toxins and metals, and liver function. When modelled against DR outcome, each group varied in performance; diabetes tests alone achieved AUC 0.72 (95\% CI: 0.68-0.76). A final clinical risk model comprising diabetes tests, BP, renal and liver function tests, haematocrit values, circulating sterols and immune markers achieved AUC 0.84 (95\% CI: 0.78-0.9) $(p=0.00013)$ (Nagelkerke $\mathrm{R}^{2}$ 0.36) (Table 5; Figure 3).

Table 5. Final clinical risk models.

\begin{tabular}{|c|c|c|c|c|}
\hline Model & Wald Test $p$-Value & McFadden $\mathbf{R}^{2}$ & Nagelkerke $R^{2}$ & AUC $(95 \%$ CI) \\
\hline Diabetes Status & $* * *$ & 0.102 & 0.142 & $0.72(0.677-0.763)$ \\
\hline Haematocrit & $* *$ & 0.007 & 0.009 & $0.57(0.539-0.601)$ \\
\hline Blood Pressure (BP) & $* * *$ & 0.017 & 0.024 & $0.586(0.554-0.618)$ \\
\hline Immune Markers & NS & 0.002 & 0.002 & $0.527(0.495-0.559)$ \\
\hline $\begin{array}{l}\text { Renal function tests } \\
\text { (renal) }\end{array}$ & $* * *$ & 0.039 & 0.054 & $0.636(0.604-0.669)$ \\
\hline $\begin{array}{l}\text { Sterols (include } \\
\text { cholesterol) }\end{array}$ & NS & 0.012 & 0.017 & $0.582(0.522-0.642)$ \\
\hline Toxins/Metals & NS & 0.029 & 0.041 & $0.589(0.478-0.7)$ \\
\hline Liver function tests & NS & 0.002 & 0.003 & $0.525(0.494-0.557)$ \\
\hline $\begin{array}{c}\text { Diabetes control + } \\
\text { BP + Renal } \\
\text { function }\end{array}$ & $* * *$ & 0.13 & 0.18 & $0.73(0.686-0.774)$ \\
\hline $\begin{array}{l}\text { Diabetes control + } \\
\mathrm{BP}+\text { renal function } \\
\text { + Haematocrit }\end{array}$ & $* * *$ & 0.135 & 0.184 & $0.737(0.694-0.78)$ \\
\hline $\begin{array}{l}\text { Diabetes control + } \\
\text { BP + renal function } \\
+ \text { Haematocrit }+ \\
\text { Sterols + Liver } \\
\text { function }\end{array}$ & $* * *$ & 0.238 & 0.315 & $0.823(0.765-0.881)$ \\
\hline All groups \pm & $* * *$ & 0.272 & 0.355 & $0.84(0.783-0.897)$ \\
\hline
\end{tabular}

Features from RandomForest ${ }^{\mathrm{TM}}$ were grouped logically based on similar function or clinical use (Table 4) and then tested independently in a univariate or multivariate regression model against DR outcome. \pm The only toxins/metal included was phosphorus (mmol/L)—others filtered out due to high missingness $(>50 \%)$, resulting in difficulty fitting model. NS, not statistically significant; ${ }^{* *}, p<0.01 ;{ }^{* * *}, p<0.001$. 

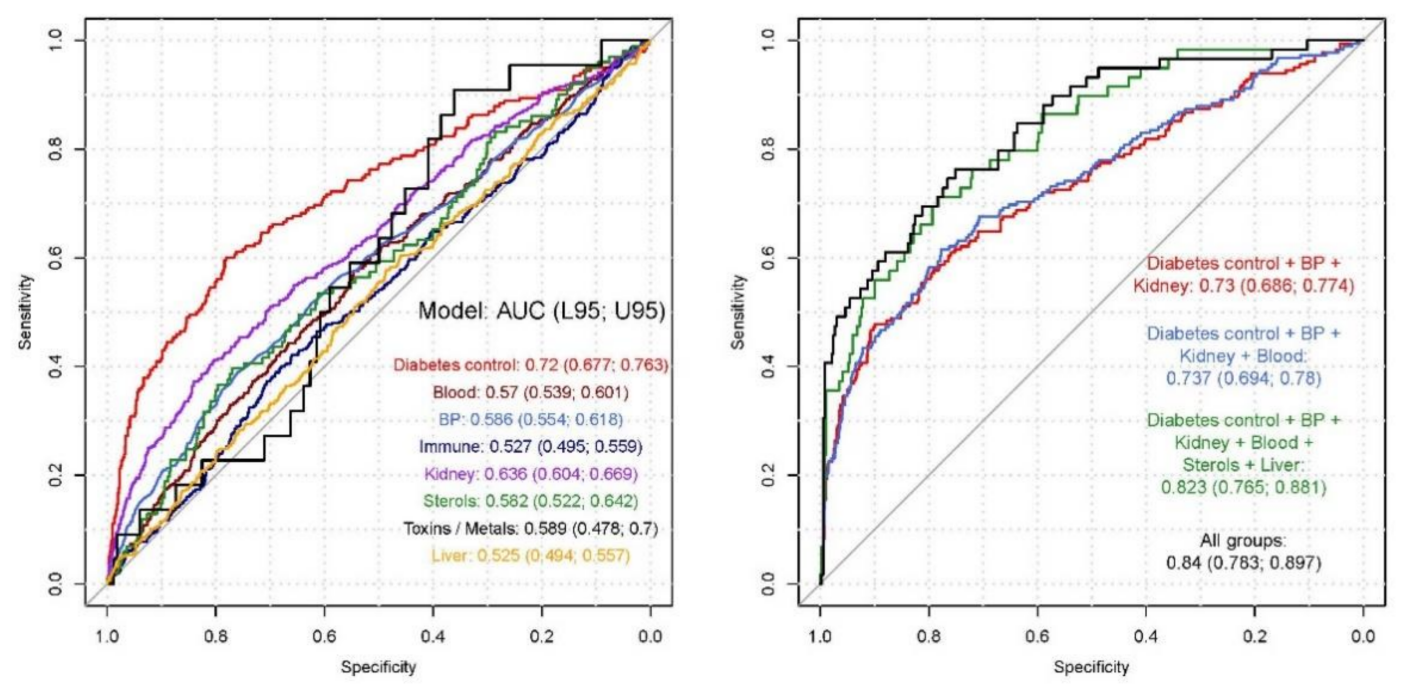

Figure 3. Final clinical risk models. Features from RandomForest ${ }^{\mathrm{TM}}$ were grouped logically based on similar function or clinical use (Table 4) and then tested independently in a univariate or multivariate regression model against diabetic retinopathy (DR) outcome. A final risk model including markers of hypertension, hypercholesterolemia, renal, and liver function tests, and haematocrit achieved AUC $0.84(0.78-0.9)$.

\section{Discussion}

This EWAS of NHANES 2007-2008 data with DR outcomes in individuals with diabetes included an unbiased feature selection approach based on a rudimentary univariate regression enabled for parallel computation, PCA, penalised regression, and RandomForest ${ }^{\mathrm{TM}}$ of a large number of laboratory parameters.

In our rudimentary approach, which is ultimately running many univariate models in a parallelised fashion, $\mathrm{HbA1c}$ was the only variable to reach statistical significance after adjustment for age, ethnicity, and diabetes duration. The relationship between $\mathrm{HbA1c}$ and DR has been explored extensively, and was selected as the strongest risk factor in every approach we undertook, with a mean decrease accuracy of $31.94 \%$ via RandomForest ${ }^{\mathrm{TM}}$. On ranking all of the risk factors and available biomarkers, hyperglycaemia not only ranked the highest, but was about three times higher than the second highest on the rank, highlighting that, in clinical practice, the patient and all healthcare professionals should pay significant emphasis on optimal control of hyperglycaemia to prevent the development and progression of DR. Although it is well known that hyperglycaemia is a risk factor of DR, DR usually develops after over a decade of diabetes, and so, this study shows that all efforts should be made to avoid patient and clinician inertia in optimally controlling hyperglycaemia over the duration of diabetes, which remains a significant challenge in most patients.

The other traditional risk factor of hypertension also remained high in the rank, but was individually not as strong as hyperglycaemia. Both the penalised regression and RandomForest ${ }^{\mathrm{TM}}$ algorithms identified an association between elevated systolic blood pressure-but not diastolic-and DR (mean decrease accuracy, $1.9 \%)$, again confirming literature [10,28-30].

When we consider the top 10 risk factors or markers associated with DR, other than hyperglycaemia, inflammatory markers and renal function markers occupy the rest of the hierarchy in the RandomForest ${ }^{\mathrm{TM}}$. Although all models show that inflammatory markers, such as CRP and white blood cell counts, are as important as renal markers, systemic anti-inflammatory agents have not shown significant effect on DR. However, monitoring these markers and treating the inflammatory focus may prevent the development and progression of DR. 
Further variables identified by both penalised regression and RandomForest ${ }^{\mathrm{TM}}$ were renal function tests including BUN, urinary albumin, potassium, osmolality, and urinary nitrate. These confirm the strong association of DR with markers of impaired kidney function.

Other known risk factors that contributed higher up in the ranking order include haematocrit (\%) and cholesterol, and these are modifiable and may offer protection.

Although $\mathrm{HbA1c}$ has the strongest association with DR, our study highlight how the addition of other clinical parameters, e.g., from renal and liver function, and haematocrit can increase the sensitivity and specificity of predicting DR outcome, with our final clinical risk model achieving AUC 0.83 (95\% CI: 0.77-0.89) ( $p=0.00012)$ (Nagelkerke $\mathrm{R}^{2} 0.33$ ), higher than any traditional diabetes control parameter in isolation or in combination.

The strengths of this study are that, in contrast to epidemiological studies that are typically conducted based on pre-conceived hypotheses and involve a single or just a few variables, the methods used in this study can be scaled to datasets of any size and therefore provide ways of working with large clinical and epidemiological datasets for the purpose of searching for novel hypotheses that could then lead to further focused investigations.

For example, the EWAS methodology and our RandomForest ${ }^{\mathrm{TM}}$ approach of non-targeted recursive feature selection also indicates a small contribution from toxins and metals, including 3-hydroxyphenanthrene, 9-hydroxyfluorene, phthalates, blood o-Xylene, and blood nitromethane. Therefore, retina may be a target tissue for environmental contamination. Some of the associations provide directions to future mechanistic research in DR. For example, we found that retinal microaneurysms (FDR-adjusted $p \leq 0.0001$ ), the most statistically significant retinal lesions in individuals with DR, is already correlated with some of the variables such as HbA1c, CRP, BUN, beryllium, and haematocrit, suggesting early effects. In contrast, increased urinary cobalt, triclosan, and barium became significant only when adjusted for duration of diabetes. Most of these parameters are also linked to risk of allergies and lung disease, an association that has not been previously explored systematically.

We observed how the PCA-based model performed slightly better than others based on AUC, with 0.796 (95\% CI: 0.761-0.832), compared to 0.74 (95\% CI: 0.72-0.77) for penalised regression and 0.71 (95\% CI: 0.65-0.77) for RandomForest ${ }^{\mathrm{TM}}$. Principal component analysis is a method that, via matrix calculations involving variance (or, more specifically, co-variance), transforms a dataset into uncorrelated PCs. Due to this uncorrelated nature and the fact that a large amount of total explained variation within a given dataset can be represented by a small number of PCs, this method works well for a complex dataset like the one in this current study. The penalised regression and RandomForest ${ }^{\mathrm{TM}}$ models gave comparable performance based on AUC, and even closer based on accuracy (78.51\% vs. $78.4 \%$, respectively). Unlike PCA, neither of these models are fundamentally based on variance (but can be indirectly influenced by it). Penalised regression with the lasso penalty is essentially a feature selection method based on logistic regression that aims to identify parameters with zero and non-zero effects; thus, the standardisation of the input parameters is important in order to mitigate effects of outliers. Penalised regression also expects that the boundary between the outcomes being measured is linear. RandomForest ${ }^{\mathrm{TM}}$, on the other hand, provides no inherent feature selection algorithm, instead assigning "importance" metrics to each parameter in the input model, metrics that can be used to set cut-offs for eliminating parameters and re-fitting the model. These models can also work with outcomes whose boundaries are non-linear in nature. It is therefore intuitive that penalised regression and RandomForest ${ }^{\mathrm{TM}}$ would result in similar performance metrics when both are correctly used. Equally, for a complex dataset, it is expected that they cannot supersede the performance of PCA.

As this is a cross-sectional study, a cause-effect relation cannot be established. Moreover, we are unable to rule out any confounding effects of any unmeasured factors. On the other hand, the main strength of the study is the use of the well characterised NHANES cohort in whom standardised protocols were used to measure laboratory parameters. We are not aware of any other association studies in DR where over 400 laboratory parameters were analysed simultaneously to develop multiple 
models. As the top variables of all four data driven agnostic models were similar, we also believe our findings are generalisable.

\section{Conclusions}

We confirm that DR is a complex disease and that the already established risk factors contribute significantly to the risk models of $\mathrm{DR}$, with $\mathrm{HbA} 1 \mathrm{c}$ being the strongest risk factor. Although our model provides an accuracy of approximately $80 \%$, it also provides mechanistic insights into future research on DR including interrogating the interaction of low-ranking risk factors with more established factors in the models and highlights need to explore epigenetic screens to gauge better how risk factors influence gene expression. Most importantly, the study reinforces the need to control known risk factors of DR, especially hyperglycaemia.

Supplementary Materials: The following are available online at http://www.mdpi.com/2077-0383/9/11/3643/s1, Table S1: Examination, demographic, and laboratory variables excluded from the study due to having zero variance, Table S2: Statistically significant principal components (PCs) associated with diabetic retinopathy (DR), Table S3: RandomForest $\mathrm{t}^{\mathrm{TM}}$-selected features for replication cohort NHANES 2005-2006, Figure S1: Laboratory variables statistically significantly associated with diabetic retinopathy, Figure S2: Inter-correlation among laboratory variables statistically significantly associated with diabetic retinopathy, Figure S3: Principal component analysis (PCA) highlights key sources of variation in the cohort.

Author Contributions: K.B., S.G., and S.S. conceived and designed the study. K.B., S.G., and Y.L. analysed the data. K.B. is the study data guarantor. All authors interpreted the results and reviewed the manuscript. K.B. had full access to all of the data and takes responsibility for the integrity of the data and the accuracy of data analysis. All authors read and approved the final manuscript.

Funding: This work was funded by Global Challenges Research Fund and UK Research and Innovation through the Medical Research Council grant number MR/P027881/1. The research was supported by the National Institute for Health Research (NIHR) Biomedical Research Centre based at Moorfields Eye Hospital NHS Foundation Trust and UCL Institute of Ophthalmology. The views expressed are those of the authors and not necessarily those of the NHS, the NIHR, or the Department of Health. The funders had no role in study design, data collection, data analysis, data interpretation, writing, editing the report, or the decision to submit for publication.

Acknowledgments: We thank the many thousands of NHANES study participants who, over the course of decades, have provided valuable information for epidemiological studies.

Conflicts of Interest: The authors declare no conflict of interest.

\section{References}

1. Feman, S.S. The natural history of the first clinically visible features of diabetic retinopathy. Trans. Am. Ophthalmol. Soc. 1994, 92, 745-773. [PubMed]

2. Chao, J.R.; Lai, M.-Y.; Azen, S.P.; Klein, A.P.; Varma, R. Retinopathy in Persons without Diabetes: The Los Angeles Latino Eye Study. Investig. Opthalmol. Vis. Sci. 2007, 48, 4019-4025. [CrossRef] [PubMed]

3. Venkatramani, J.; Mitchell, P. Ocular and systemic causes of retinopathy in patients without diabetes mellitus. BMJ 2004, 328, 625-629. [CrossRef] [PubMed]

4. Gupta, A.; Singh, R.; Ramasamy, K.; Abraham, C.; Gupta, V. Diabetic retinopathy: An update. Indian J. Ophthalmol. 2008, 56, 179. [CrossRef]

5. Wang, W.; Lo, A. Diabetic Retinopathy: Pathophysiology and Treatments. Int. J. Mol. Sci. 2018, $19,1816$. [CrossRef]

6. Yau, J.W.; Rogers, S.L.; Kawasaki, R.; Lamoureux, E.L.; Kowalski, J.W.; Bek, T.; Chen, S.-J.; Dekker, J.M.; Fletcher, A.E.; Grauslund, J.; et al. Global Prevalence and Major Risk Factors of Diabetic Retinopathy. Diabetes Care 2012, 35, 556-564. [CrossRef]

7. Heintz, E.; Wiréhn, A.B.; Peebo, B.B.; Rosenqvist, U.; Levin, L.A. Prevalence and healthcare costs of diabetic retinopathy: a population-based register study in Sweden. Diabetologia 2010, 53, 2147-2154. [CrossRef]

8. Group UPDSU. Intensive blood-glucose control with sulphonylureas or insulin compared with conventional treatment and risk of complications in patients with type 2 diabetes (UKPDS 33). Lancet 1998, 352, 837-853. [CrossRef] 
9. Chatziralli, I. The Role of Dyslipidemia Control in the Progression of Diabetic Retinopathy in Patients with Type 2 Diabetes Mellitus. Diabetes Ther. 2017, 8, 209-212. [CrossRef]

10. Liu, L.; Quang, N.D.; Banu, R.; Kumar, H.; Tham, Y.-C.; Cheng, C.-Y.; Wong, T.Y.; Sabanayagam, C. Hypertension, blood pressure control and diabetic retinopathy in a large population-based study. PLoS ONE 2020, 15, e0229665. [CrossRef]

11. Chen, X.; Meng, Y.; Li, J.; She, H.; Zhao, L.; Zhang, J.; Peng, Y.; Shang, K.; Zhang, Y.; Gu, X.; et al. Serum uric acid concentration is associated with hypertensive retinopathy in hypertensive chinese adults. BMC Ophthalmol. 2017, 17, 83. [CrossRef] [PubMed]

12. Luo, B.-A.; Gao, F.; Qin, L.-L. The Association between Vitamin D Deficiency and Diabetic Retinopathy in Type 2 Diabetes: A Meta-Analysis of Observational Studies. Nutrients 2017, 9, 307. [CrossRef] [PubMed]

13. Kong, X.; Wang, J.; Gao, G.; Tan, M.; Ding, B.; Li, H.; Ma, J.-H. Association between Free Thyroxine Levels and Diabetic Retinopathy in Euthyroid Patients with Type 2 Diabetes Mellitus. Endocr. Res. 2020, 45, 111-118. [CrossRef] [PubMed]

14. Merin, S.; Freund, M. Retinopathy in Severe Anemia. Am. J. Ophthalmol. 1968, 66, 1102-1106. [CrossRef]

15. Khan, A.A.; Rahmani, A.H.; Aldebasi, Y.H. Diabetic Retinopathy: Recent Updates on Different Biomarkers and Some Therapeutic Agents. Curr. Diabetes Rev. 2018, 14, 523-533. [CrossRef]

16. Moriya, T.; Tanaka, S.; Kawasaki, R.; Ohashi, Y.; Akanuma, Y.; Yamada, N.; Sone, H.; Yamashita, H.; Katayama, S. Diabetic Retinopathy and Microalbuminuria Can Predict Macroalbuminuria and Renal Function Decline in Japanese Type 2 Diabetic Patients. Diabetes Care 2013, 36, 2803-2809. [CrossRef]

17. Chen, Y.-H.; Chen, H.-S.; Tarng, D.-C. More Impact of Microalbuminuria on Retinopathy Than Moderately Reduced GFR Among Type 2 Diabetic Patients. Diabetes Care 2012, 35, 803-808. [CrossRef]

18. Lim, L.S.; Tai, E.S.; Mitchell, P.; Wang, J.J.; Tay, W.T.; Lamoureux, E.; Wong, T.Y. C-reactive Protein, Body Mass Index, and Diabetic Retinopathy. Investig. Opthalmol. Vis. Sci. 2010, 51, 4458-4463. [CrossRef]

19. Raymond, N.T.; Varadhan, L.; Reynold, D.R.; Bush, K.; Sankaranarayanan, S.; Bellary, S.; Barnett, A.H.; Kumar, S.; O'Hare, J.P. Higher prevalence of retinopathy in diabetic patients of South Asian ethnicity compared with white Europeans in the community: A cross-sectional study. Diabetes Care 2009, 32, 410-415. [CrossRef]

20. Spanakis, E.K.; Golden, S.H. Race/Ethnic Difference in Diabetes and Diabetic Complications. Curr. Diabetes Rep. 2013, 13, 814-823. [CrossRef]

21. Wong, T.Y.; Klein, R.; Islam, F.M.A.; Cotch, M.F.; Folsom, A.R.; Klein, B.E.K.; Sharrett, A.R.; Shea, S. Diabetic Retinopathy in a Multi-ethnic Cohort in the United States. Am. J. Ophthalmol. 2006, 141, 446-455.e1. [CrossRef] [PubMed]

22. McGinnis, D.P.; Brownstein, J.S.; Patel, C.J. Environment-Wide Association Study of Blood Pressure in the National Health and Nutrition Examination Survey (1999-2012). Sci. Rep. 2016, 6, 30373. [CrossRef] [PubMed]

23. Zhuang, X.; Guo, Y.; Ni, A.; Yang, D.; Liao, L.; Zhang, S.; Zhou, H.; Sun, X.; Wang, L.; Wang, X.; et al. Toward a panoramic perspective of the association between environmental factors and cardiovascular disease: An environment-wide association study from National Health and Nutrition Examination Survey 1999-2014. Environ. Int. 2018, 118, 146-153. [CrossRef] [PubMed]

24. Hall, M.A.; Dudek, S.M.; Goodloe, R.; Crawford, D.C.; Pendergrass, S.A.; Peissig, P.; Brilliant, M.; McCarty, C.A.; Ritchie, M.D. Environment-wide association study (EWAS) for type 2 diabetes in the Marshfield Personalized Medicine Research Project Biobank. Pac. Symp. Biocomput. 2014, 2014, 200-211.

25. Patel, C.J.; Rehkopf, D.H.; Leppert, J.T.; Bortz, W.M.; Cullen, M.R.; Chertow, G.M.; Ioannidis, J.P. Systematic evaluation of environmental and behavioural factors associated with all-cause mortality in the United States National Health and Nutrition Examination Survey. Int. J. Epidemiol. 2013, 42, 1795-1810. [CrossRef]

26. Nembrini, S.; König, I.R.; Wright, M.N. The revival of the Gini importance? Bioinformatics 2018, 34, 3711-3718. [CrossRef]

27. Benjamini, Y.; Hochberg, Y. Controlling the False Discovery Rate: A Practical and Powerful Approach to Multiple Testing. J. R. Stat. Soc. Ser. B (Methodol.) 1995, 57, 289-300. [CrossRef]

28. Stratton, I.M.; Kohner, E.M.; Aldington, S.J.; Turner, R.C.; Holman, R.R.; Manley, S.E.; Matthews, D.R. UKPDS 50: Risk factors for incidence and progression of retinopathy in Type II diabetes over 6 years from diagnosis. Diabetologia 2001, 44, 156-163. [CrossRef] 
29. Klein, R. (Ronald) Blood pressure control and diabetic retinopathy. Br. J. Ophthalmol. 2002, 86, $365-367$. [CrossRef]

30. Zheng, Y.; Lamoureux, E.L.; Lavanya, R.; Wu, R.; Ikram, M.K.; Wang, J.J.; Mitchell, P.; Cheung, N.; Aung, T.; Saw, S.-M.; et al. Prevalence and risk factors of diabetic retinopathy in migrant Indians in an urbanized society in Asia: The Singapore Indian eye study. Ophthalmology 2012, 119, 2119-2124. [CrossRef]

Publisher's Note: MDPI stays neutral with regard to jurisdictional claims in published maps and institutional affiliations.

(C) 2020 by the authors. Licensee MDPI, Basel, Switzerland. This article is an open access article distributed under the terms and conditions of the Creative Commons Attribution (CC BY) license (http://creativecommons.org/licenses/by/4.0/). 